\title{
EXPERIMENTAL STUDY FOR RETAINING WALLS USING PILE COUNTERMEASURE AGAINST EARTHQUAKES
}

\author{
ENAYAT MALLYAR \& KAKUTA FUJIWARA \\ Department of Civil Engineering, Tokai University, Japan
}

\begin{abstract}
In many coastal areas, port facilities such as retaining walls were highly damaged due to earthquakes. Deformation of retaining walls is one of the primary sources of damage in the port facilities. The liquefaction of the backfill causes a large lateral force to the walls, which is triggered by the wall's displacement. The retaining wall has been constructed widely and required to be resistant during earthquake motion. Several effective countermeasures to mitigate liquefaction have been proposed. Using piles as a countermeasure nearby the retaining walls has been proposed that the displacement of the retaining walls could be reduced. However, the high rigid material pile is required high cost. Therefore, the authors conducted a series of shaking model tests in three cases with different high rigid and low rigid types of piles to investigate the effectiveness of piles against the deformation of the retaining wall. The deformation of the retaining wall, caused by liquefied soil, was obtained by the sensors measurement and it is shown that even mix (high rigidity and low rigidity) piles in the backfill could effectively reduce the wall displacement.
\end{abstract}

Keywords: retaining wall, pile countermeasure, shaking table test, earthquake.

\section{INTRODUCTION}

Soil liquefaction brought severe damage to structures in many past earthquakes. Port structures have often suffered damages in strong earthquakes. Loose sandy soil behind retaining walls are susceptible to liquefaction. The liquefaction of the backfill causes a large lateral force to the wall which is cause the deformation and extensive damage to the retaining walls. During several destructive earthquakes such as the 1983 Nihonkai-Chubu and the 1995 Hyogoken-Nambu (Kobe) earthquake [1] a large number of retaining walls, including quay walls, were damaged. Although extensive analytical and experimental studies (using a vibration table and centrifuge model) have been conducted on retaining walls behavior undergoing lateral flow of liquefied soil.

Several countermeasure methods against liquefaction have been proposed such as soil improvement techniques represented by sand compaction, stone column and seismic reinforcement using steel piles techniques, can reduced the generated lateral force of liquefied soil. One of the effective methods is the deterrent pile method [2], where steel piles are installed in the backfill, distributive and not connected with retaining walls. It has been verified through centrifuge model tests that these piles prevent soil from flowing laterally and interdict the increasing excess pore water pressure of soil near the piles. However, still it is needed to investigate on different types and numbers of piles because using many steel piles is required high material cost. Steel pile has high rigidity, but it is expensive, other low rigid materials are almost inexpensive. Therefore, the authors conducted series of shaking model tests on different types of piles in the back fill of a retaining wall. 


\section{TEST CONDITION}

\subsection{Device and soil}

The model was geometrically scaled to approximately $1 / 20-1 / 30$ of the actual structure, as shown in Fig. 1. The model was built in a rigid container with transparent side wall. Dimension of the container were $0.84 \times 0.60 \times 0.44 \mathrm{~m}^{3}$. The rigid container filled in two soil layers; the bottom layer was filled and compacted as a dense layer and one half of the second layer made as a loose layer and the other half was filled with water. The soil for both layers was Toyoura sand $\left(\mathrm{e}_{\max }=0.927, \mathrm{e}_{\min }=0.625, \rho_{\mathrm{s}}=2.64 \mathrm{~g} / \mathrm{cm}^{3}\right)$. The experiments were conducted in three cases. Case A is no countermeasure case (without pile), as shown in Fig. 1(a). In the Case B ten acryl piles (high rigidity) installed in three rows as shown in Fig. 2(a). In the Case $C$ seven acryl piles (high rigidity) installed in first and second row from the wall and three rubber piles (low rigidity) installed in third row, as shown in Fig. 2(b). The three cases mitigation strategy and the dense and loose layers properties shown in Table 1.

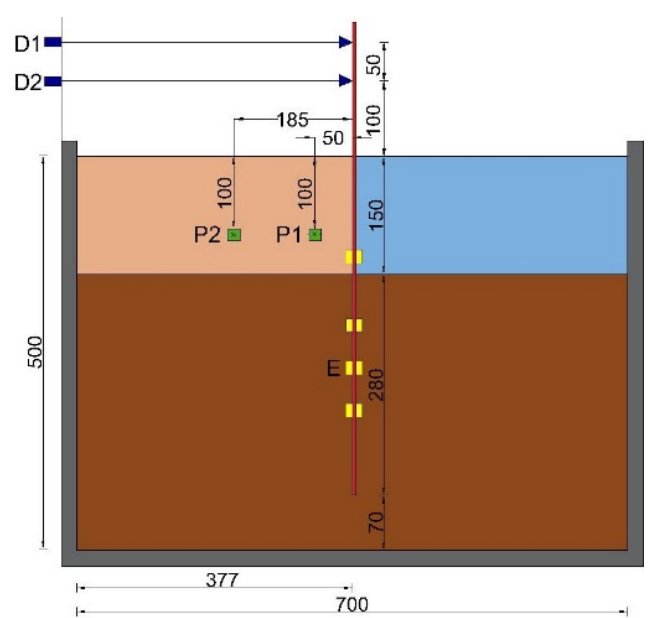

(a)

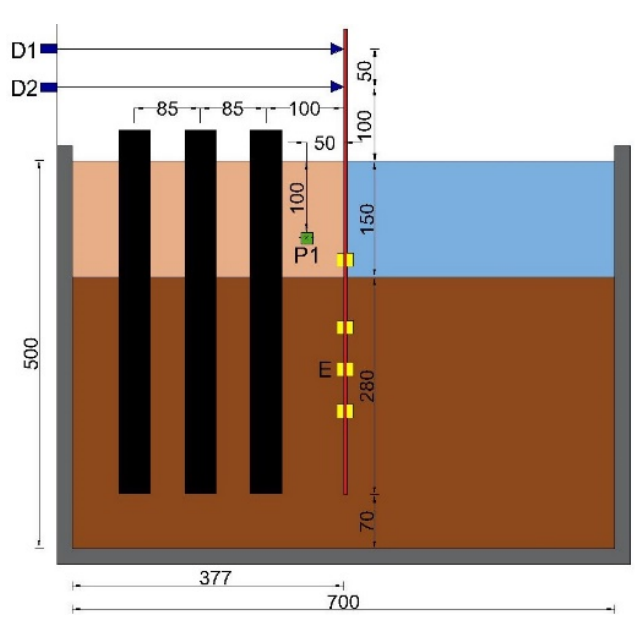

(b)

Figure 1: Test model cross section. (a) Case A; and (b) Cases B and C. Note: D1, D2 = Displacement sensor; $\mathrm{P} 1, \mathrm{P} 2=$ Water pressure sensor; $\mathrm{E}=$ Strain gauge sensor.

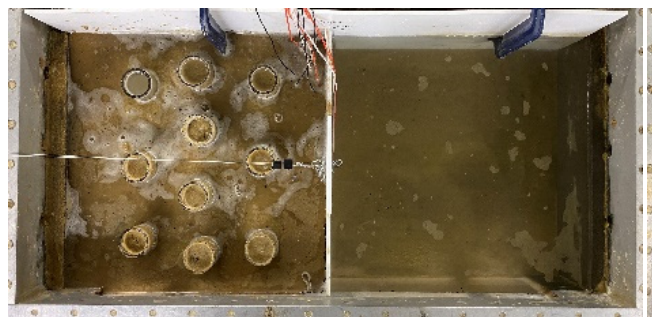

(a)

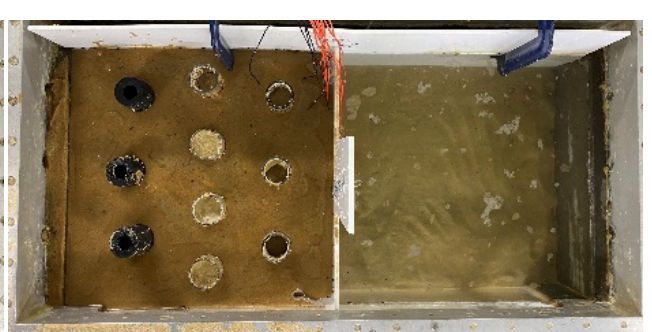

(b)

Figure 2: The model top view. (a) Case B; and (b) Case C. 
Table 1: Summarizes the features of each experiment.

\begin{tabular}{|l|c|c|c|c|l|}
\hline \multirow{2}{*}{ Cases } & \multicolumn{2}{|c|}{ Dense layer } & \multicolumn{2}{c|}{ Loose layer } & Mitigation strategy \\
\cline { 2 - 6 } & $\operatorname{Dr}(\%)$ & $\gamma_{\mathrm{t}}\left(\mathrm{kN} / \mathrm{m}^{3}\right)$ & $\operatorname{Dr}(\%)$ & $\gamma_{\mathrm{t}}\left(\mathrm{kN} / \mathrm{m}^{3}\right)$ & \\
\hline Case A & 94 & 17.0 & 40 & 14.3 & No countermeasure \\
\hline Case B & 97 & 17.1 & 43 & 15.2 & 10 Acryl piles \\
\hline Case C & 91 & 17.3 & 44 & 15.0 & 7 Acryl and 3 rubber piles \\
\hline
\end{tabular}

\subsection{Materials properties}

In this experimental model were used two types of piles, acryl pile represents rigid feature and rubber pile represents low rigidity as shown in Figs 3 and 4. Both types of pile with $40 \mathrm{~mm}$ diameter and $450 \mathrm{~mm}$ length were installed in backfill of the wall. An acryl plate with $5 \mathrm{~mm}$ thickness and $470 \mathrm{~mm}$ length as a retaining wall was located inside the dense layer, $70 \mathrm{~mm}$ upper than the model box base.
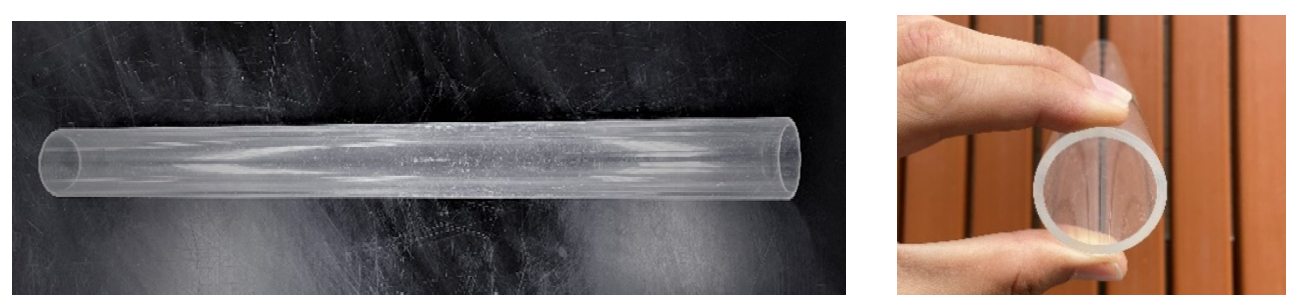

Figure 3: Acryl pile used as a high-rigidity pile.
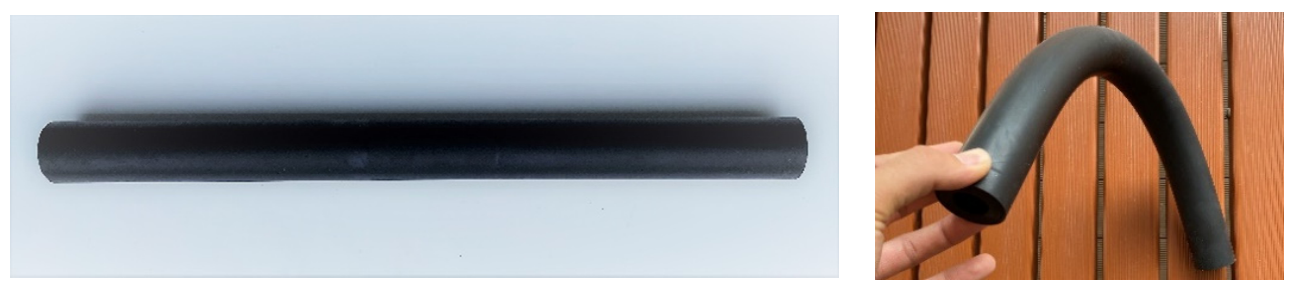

Figure 4: Rubber hose used as a low-rigidity pile.

\subsection{Input motion}

The input motion was a sine wave of frequency $3 \mathrm{~Hz}$ for 5 seconds. The sine wave was used as a simple motion. Considering actual field, the characters of the wave; duration, frequency and wave shape should be examined in further study.

Three motions with intervals were given to the device for each case. The maximum acceleration for each motion were $0.5,1.0$ and $2.0 \mathrm{~m} / \mathrm{s}^{2}$. In this paper, the authors regarded $0.5 \mathrm{~m} / \mathrm{s}^{2}$ as a weak motion, $1 \mathrm{~m} / \mathrm{s}^{2}$ as a medium motion and $2 \mathrm{~m} / \mathrm{s}^{2}$ as a strong motion. The acceleration of $0.5 \mathrm{~m} / \mathrm{s}^{2}$ is a weak motion as small as the soil had the excess pore water pressure. The authors increased the acceleration as 1.0 and $2.0 \mathrm{~m} / \mathrm{s}^{2}$ to investigate the effectiveness against the acceleration of earthquake. This model test was equipped with three types of sensors, two displacement sensors sticked on $100 \mathrm{~mm}$ and $150 \mathrm{~mm}$ upper than top 
of wall, eight strain gauge sensors sticked on both sides of the wall, and two water pressure sensors that one of them located between wall and piles and another one located between piles in the liquefiable layer as shown in Fig. 1.

\section{TEST RESULT}

The model was shaken under three input motions. Based on observations, the soil seems to be liquefied, that caused the wall deformations. Detailed information are discussed in this section.

\subsection{Displacement}

The residual horizontal displacement at the D point (D point presented the top of wall which is derived by two displacement sensors D1 and D2) for all cases shown in Fig. 5(a). The acceleration of zero means before shaking state. The larger the acceleration became, the larger the displacement became, for all cases. Fig. 5(a) shows that the displacement of Case $\mathrm{B}$ was smallest among all cases. The displacements for Cases B and C are smaller than that of Case A, which means the pile countermeasures were effective for wall displacement. Additionally, the displacements for Cases B and C are not same, which means the structure of Case B was more effective than that of Case $\mathrm{C}$ though the difference was small. The result indicates the displacement for Case $\mathrm{C}$ is smaller than Case $\mathrm{A}$, which means even the mix piles countermeasure was effective against wall deformation.

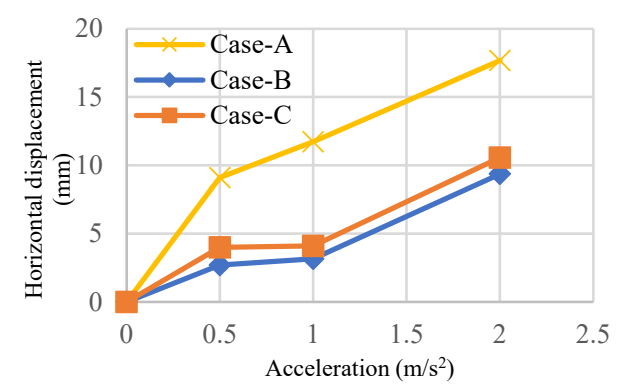

(a)

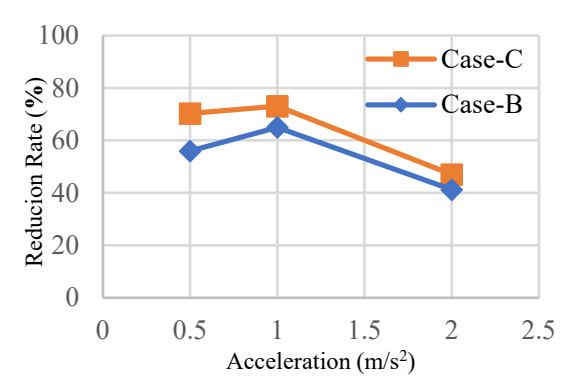

(b)

Figure 5: Horizontal displacement of retaining wall. (a) Displacement at point D; and (b) Reduction rate.

The reduction rate means that the displacements of Cases $\mathrm{B}$ and $\mathrm{C}$ are divided by the displacement of Case A for each acceleration, as shown in Fig. 5(b). The reduction rates of Case B and Case C were lager than $50 \%$ for the weak and medium motion $\left(0.5 \mathrm{~m} / \mathrm{s}^{2}\right.$ and $1 \mathrm{~m} / \mathrm{s}^{2}$ ). On the other hands, the reduction rate of Case B and Case C were less than $50 \%$ for the strong motion $\left(2 \mathrm{~m} / \mathrm{s}^{2}\right)$. These results state that the pile countermeasures are more effective for weak and medium motions, even soft pile is effective to reduce the wall displacement. Fig. 5(b) indicates that the reduction rate of Case B was more than that of Case $\mathrm{C}$ though the difference was small. 


\subsection{Bending strain}

The residual bending strain along the retaining wall for three motions in all cases are depicted in Fig. 6. The strain gauge sensor at the height of $170 \mathrm{~mm}$ for Case A did not work. In these figures the horizontal line at the height of $350 \mathrm{~mm}$ shows the border between dense and loose soil layer. Fig. 6 indicates the wall was deformed toward the water side due to the liquefaction of soil. The bigger motion caused the bigger bending strain on the retaining wall among all cases. Through the three motions, the bending strain of Case B was smallest among three cases. The pile countermeasure (Case B) could reduce the wall deformation in spite of the difference of acceleration. This result matches to the result of the displacement indicated in the previous section. On the other hands, the strain for Case $\mathrm{C}$ was almost same as that for Case A. This result doesn't match to the result of the displacement indicated in the previous section. As the strain for Case A has a lack of data that mentioned above, the author continues to investigate this result.

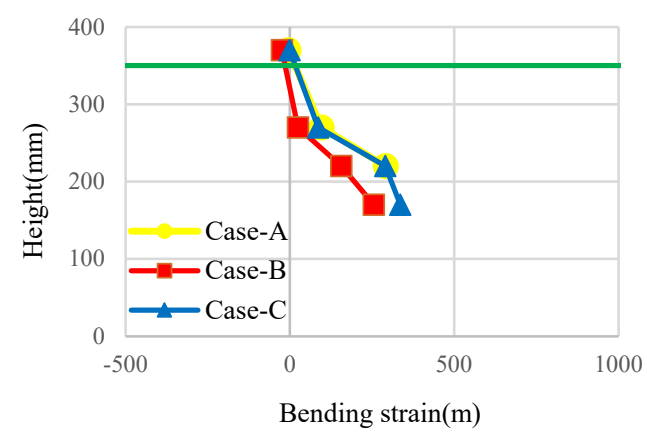

(a)

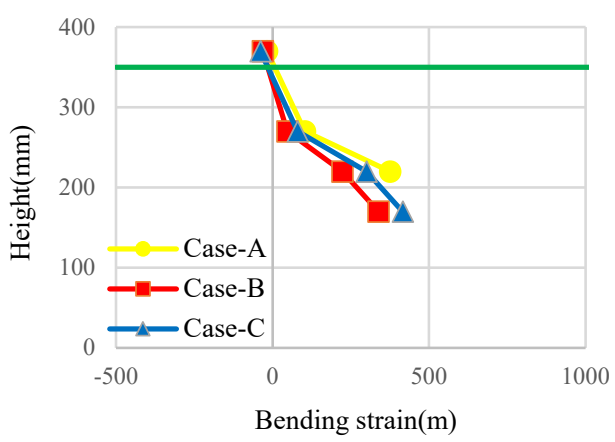

(b)

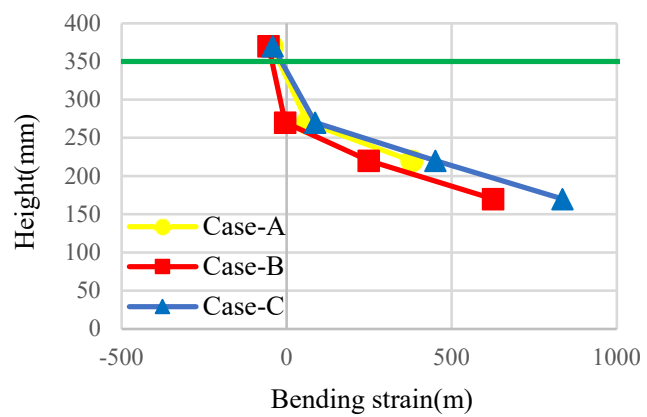

(c)

Figure 6: The residual bending strain along the retaining wall. (a) 50 gal; (b) 100 gal; and (c) 200 gal.

\subsection{Water pressure}

When the earthquake occurs, water pressure increases, and soil gets shear deformation. Shear deformation makes soil liquefied. Figs 7 and 8 show the excess pore water pressure at point P1 and P2 for all cases, respectively. According to the acceleration time histories of excess pore water pressure, high pressure developed at the early stage of shaking. Fig. 7(a) and Fig. 
8(a) indicate the excess pore water pressure increased due to the motion that means soil liquefaction. After that, the excess pore water pressure was converged to a constant value. Fig. 7(b), (c) and Fig. 8(b), (c) state the excess pore water pressure started from the remaining value in previous motion, respectively. Compering among 50-200 gal at P1 and P2, it can be seen, as the acceleration got bigger the excess pore water pressure became bigger. The excess pore water pressure were less at P1 than $\mathrm{P} 2$. This is because the sand near to the wall was expanded by the deformation of the wall. Fig. 7(a)-(c) and Fig. 8(a)-(c) show that the excess pore water pressure in Case B and Case C were smaller than that in Case A. The result illustrates that the acryl (high rigidity) piles and mix (high rigidity and low rigidity) piles prevented the shear deformation of soil, that caused the prevention of water pressure increment. The reduction of water pressure caused the reduction of the wall displacement.

\section{CONCLUSION}

For the reduction of the material cost of retaining wall countermeasure, the authors proposed the mix pile countermeasure which consists of high rigidity and low rigidity piles. The authors conducted model tests; three types of motions (weak, medium, and strong) are given continuously with interval to three model cases (no pile, high rigidity piles, mix of high and low rigidity piles). Through the model tests, following conclusions are gained.

1. The sand was liquefied by the motion, which caused wall deformation. The larger the acceleration, the larger the wall displacement became with or without piles.

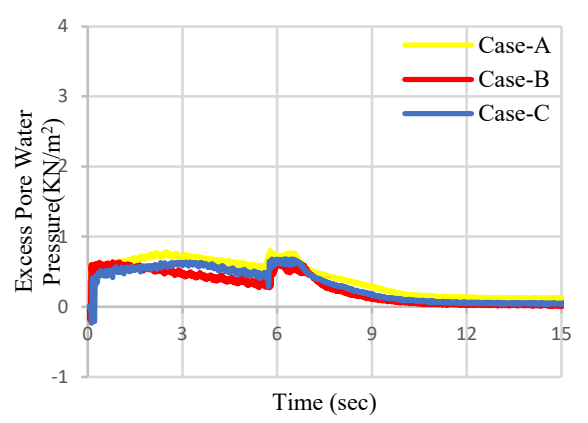

(a)

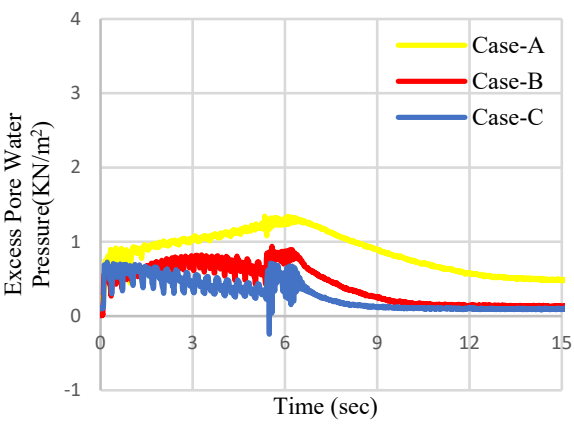

(b)

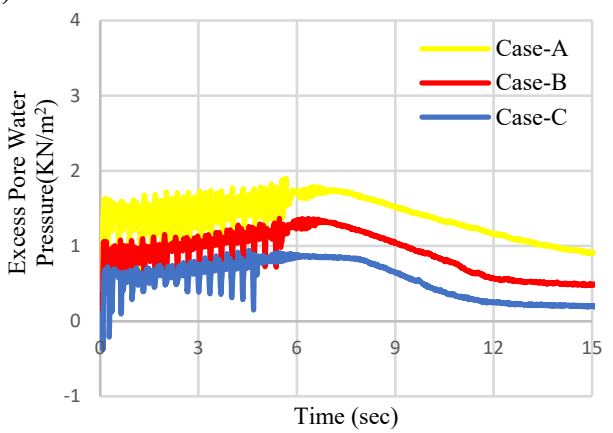

(c)

Figure 7: The time histories of excess pore water pressure of P1. (a) 50 gal; (b) 100 gal; and (c) 200 gal. 


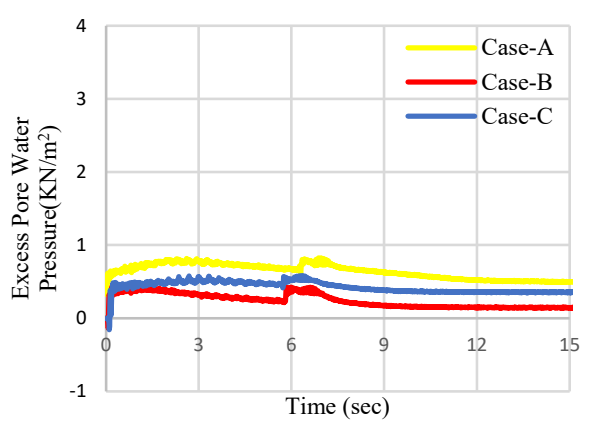

(a)

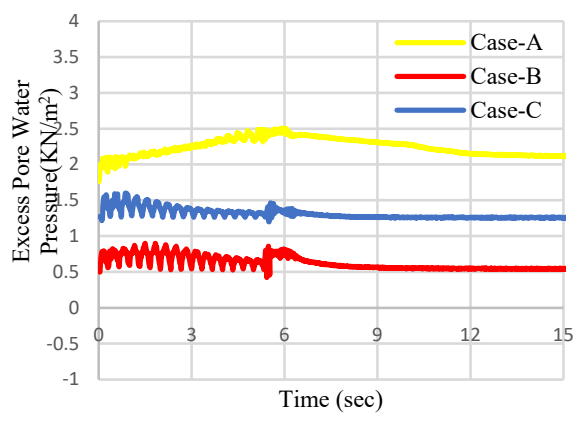

(b)

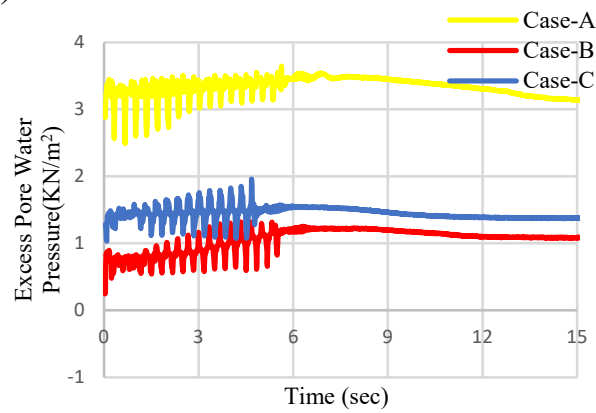

(c)

Figure 8: The time histories of excess pore water pressure of P2. (a) 50 gal; (b) 100 gal; and (c) 200 gal.

2. In high rigidity piles case, the wall displacement was $47-73 \%$ reduced compared to no pile case. In addition, even in mix pile case, the wall displacement was $40-65 \%$ reduced compared to no pile case. There is small difference between high rigidity piles and mix piles countermeasure.

3. Both high rigidity piles and mix piles were more effective against the wall deformation under weak motion than that strong motion.

4. The piles as a countermeasure could prevent water pressure from increasing and prevent the shear deformation of soil.

5. To find out the accurate effectiveness of rubber pile, we need more investigation by considering two rows and one row of piles and changing the number of the rubber piles.

\section{ACKNOWLEDGEMENT}

This work was supported by JSPS KAKENHI Grant Number 19K15265. We would like to express our thanks to JSPS.

\section{REFERENCES}

[1] Tokimatsu, K. \& Asaka, Y., Effects of liquefaction-induced ground displacements on pile performance in the 1995 Hyogoken-Nambu earthquake. Special Issue of Soils and Foundation, pp. 163-177, 1998. 
[2] Hamada, M. \& Higuchi, S., Development of countermeasures for lateral flow of liquefied ground. Journal of Japan Society of Civil Engineers, Ser. A1 (Structural Engineering and Earthquake Engineering (SE/EE)), 66(1), pp. 84-94, 2010.

[3] Fujiwara, K., Numerical study on pile countermeasure against liquefaction behind retaining wall. 29th International Ocean and Polar Engineering Conference. OnePetro, 2019.

[4] Motamed, R. \& Towhata, I., Mitigation measures for pile groups behind quay walls subjected to lateral flow of liquefied soil: Shake table model tests. Soil Dynamics and Earthquake Engineering, 30(10), pp. 1043-1060, 2010.

[5] Ogata, T., Disaster management in Japan. JMA-PhRMA Joint Symposium Conferences, 59(1), pp. 27-30, 2016. https://www.pwri.go.jp/eng/ujnr/joint/41/paper/17_Ikeuchi.pdf.

[6] Miura, K., Kohama, E., Inoue, K., Ohtsuka, N., Sasajima, T. \& Yoshida N., Behavior of gravity type quay wall during earthquake regarding dynamic interaction between caisson and backfill during liquefaction. Proceedings of the 12th World Conference on Earthquake Engineering, 7, p. 1737, 2000.

[7] Kohama, E., Sugano, T., Shiozaki, Y. \& Mitoh, M., Dynamic behaviour of a steel sheet pile quay wall in the full scale experiment with regard to liquefaction. Proceedings of the 13th World Conference on Earthquake Engineering, Vancouver, BC, Canada, 2004. 